\title{
MODELING MULTIMEDIA DISPLAYS USING ACTION BASED TEMPORAL LOGIC
}

\author{
Graciela González \\ Sam Houston State University \\ Dept.of Mathematics, Computer Science, and Statistics \\ Huntsville, TX, U.S.A. \\ csc.ghg@shsu.edu
}

\author{
Chitta Baral \\ Arizona State University \\ Department of Computer Science and Engineering \\ Tempe, AZ 85287-5406, U.S.A. \\ chitta@asu.edu
}

\author{
Peter A. Cooper \\ Sam Houston State University \\ Dept.of Mathematics, Computer Science, and Statistics \\ Huntsville, TX, U.S.A. \\ csc pac@shsu.edu
}

\begin{abstract}
We present a metalanguage, named Alan that can be used to model dynamic multimedia displays, particularly those that display multimedia database query results. Alan is an action language that uses temporal logic to model nonMarkovian systems. We show how it can be used for specifying the behavior of fairly complex dynamic multimedia display systems, modeling all graphical user interface elements on the display plus the effects of actions and of the passage of time on media such as video and audio.
\end{abstract}

Keywords: Multimedia, modeling, semantics, temporal logic, action languages, presentations, temporal presentations. 


\section{INTRODUCTION}

The display of multimedia documents and presentations is usually built around common graphical user interface elements, such as buttons, checkboxes, lists, images, video and audio players, to name a few. The relationship among the different elements and the data that is displayed on them can be either pre-built or dynamically allocated in response to user queries or requests. Dynamically constructed multimedia documents that allow the user to interact with the data are the primary concern of this paper.

The semantics of a multimedia document are not obvious. There might be constraints on the behavior of elements that are dependant on not only the most recent action, but past actions as well. For example, consider a display that contains a video. By analyzing the layout, all we can say is where the video will play, but not for how long, or starting from which point in time. These two conditions might be determined by user actions that occur just before the video plays, or while it plays. A formal semantics or a formal model provides a means to unambiguously express such behavior.

We propose a way to model the behavior of multimedia displays, particularly those derived from database queries, using a new action language with temporal extensions we have called Alan. It allows enough descriptive power to express the behavior of complex, dynamic multimedia displays, modeling user-initiated actions and system actions, temporal presentations, triggers, and the behavior of data elements that span over time, like video and audio files.

The specification of the layout is not our primary concern. Any display specification language or formalism can be used to define the layout (positioning) of the elements on the display, or even natural language.

\section{THE LANGUAGE ALAN}

Action languages are logical formalisms used to represent dynamic environments in which actions affect the state of the environment. We refer to the language we present as Alan, short for Action LAnguage for Nonmarkovian domains. It is an action language that can model systems where the Markov restriction does not necessarily hold. The Markov restriction states that the future state of the world is independent of the past states given the current state (Shanmugan \& Breipohl, 1988). If the Markov restriction holds, conditions placed on the executability of actions or on their effects can only be based on the current state of the world.

Formulas in Alan are used to determine whether an action is possible or not. Unlike in traditional action languages, formulas in Alan can incorporate 
past temporal operators such as "previously" or "lasttime" to reference points in the past. Therefore, Alan allows the definition of systems where the future state of the world depends not only on the current state, but also on past states of the world. That is, of non-Markovian systems.

Even though Alan uses the syntax of action description languages $A$ and $L_{o}$ presented in (Baral et al., 1997) and extended in (Watson 1999), its semantics are radically different, since $A$ and $L_{o}$ can only define Markovian systems. In this section we present an overview of Alan, enough to understand how it is used to model dynamic displays.

\subsection{Basic notions}

In Alan, we assume an alphabet for the language consisting of two disjoint, nonempty sets of symbols: a set $\mathbf{F}$ of fluents and a set $\mathbf{A}$ of actions.

As in all action languages, a fluent is a proposition whose truth-value might vary from one state of the world to the next. A literal is a fluent $f$ or its negation $\neg f$. A state consists of a set of fluents that give a snapshot of the state of the world at some point in time. Consecutively indexed letters $s$ denote states. Actions change the truth-value of fluents, determining new states. Actions can be elementary or compound. A compound action is a set of elementary actions that occur concurrently.

An alternating sequence of states and actions is called a path. A path that conforms to certain properties is called a trajectory. It is the means to represent the flow of time and the key to interpret formulas and propositions.

\subsection{Formulas, Propositions, and Action Description}

The simplest construct in Alan is a formula, which can be an action, state, and temporal formula. We refer to them indistinctively as formulas when something applies to all or when the type of formula is clear by context. An action formula is of the form "occurs $a$ ", where $a$ is a compound or elementary action from A. A state formula is an action formula or a literal, or, if $p, p_{1}$, and $p_{2}$, are state formulas, their negation, conjunction, or disjunction, written $\neg p, p_{1} \wedge p_{2}$ and $p_{1} \vee p_{2}$ are also state formulas.

A state formula can also be considered a temporal formula. In addition, if $p, p_{1}$ and $p_{2}$ are formulas, then (1) lasttime $p$, (2) previously $p$ (3) $p_{1}$ before $p_{2}$, and (3) $p_{1}$ since $p_{2}$ are temporal formulas. The negation, conjunction, and disjunction of temporal formulas are considered temporal formulas. The temporal operators (1) to (4) above can also be used to write the temporal connectives always and never, which we will use as well:

always $p$ ( $\boldsymbol{p}$ was true always in the past) can be written $\neg$ previously $\neg p$ never $\boldsymbol{p}$ ( $p$ has never been true in the past) can be written $\neg$ previously $\mathbf{p}$ 
Since we can express implication $\mathrm{p}_{1} \rightarrow \mathrm{p}_{2}$ for any pair of formulas $\mathrm{p}_{1}$ and $\mathrm{p}_{2}$ in terms of ' $\neg$ ' and ' $\wedge$ ' as $\neg\left(\mathrm{p}_{1} \wedge \neg \mathrm{p}_{2}\right)$, we will also use implication freely.

Formulas are used to form propositions, which express the conditions upon which actions are performed, and the effects of those actions.

If $a$ is an action, $a_{e}$ is an elementary action, $\lambda$ is a literal, and $p$ is a formula, propositions take one of four forms in Alan (1) $\lambda$ if $p$, called a static proposition (2) $a_{e}$ causes $\lambda$ if $p$, called a causal proposition, (3) impossible $a$ if $p$, called an impossibility proposition, and (4) defined $\lambda$ if $p$, called a definition proposition.

A collection of propositions is called an action description.

The following example illustrates the use of Alan to describe a general non-Markovian system.

Example 1. Upon receiving a check, a credit card company deposits it, and gives credit to the customer. However, if the check is returned (bounces), then they deposit it again, without giving credit twice. If it bounces a second time, then the credit is reversed.

In Alan, these facts can be expressed as follows:

DEPOSIT causes CREDITED if $\neg$ previously occurs DEPOSIT

$\neg$ CREDITED if occurs BOUNCES ^ previously occurs BOUNCES

\subsection{Interpreting formulas}

An action description in a Markovian language defines a collection of acceptable paths or "possible trajectories" of the corresponding domain. However, given that Alan is non-Markovian, auxiliary definitions are needed, which are presented in (Gonzalez 2000) in the full semantics of the language. In a nutshell, the full semantics define when an action is executable, the effects of actions, the closure of a path with respect to an action description, and the set of possible trajectories in the non-Markovian setting.

Suffice here to say that in general, formulas have a truth-value only over a path $\pi$. Intuitively, this is because formulas might include temporal connectives such as "previously" that refer to properties of past states or properties that hold throughout past states. Given a path $\pi=\left\langle s_{0}, a_{l}, s_{1}, \ldots, a_{n}\right.$, $s_{n}>$, in Alan, by $\pi_{\mathrm{j}}, \mathrm{j} \leq \mathrm{n}$, we denote $\left\langle s_{0}, a_{l}, s_{l}, \ldots ., a_{j}, s_{j}>\right.$, that is, the suffix of $\pi$ that ends at state $s_{\mathrm{j}}$. Given a path $\pi$ of length $\mathrm{n}$, an action $a$, and state formulas $p, q$, we determine the truth of formulas as follows:

1. A literal $\lambda$ is true in $\pi_{\mathrm{n}}$ if $\lambda \in \mathrm{s}_{\mathrm{n}}$.

2. occurs $a$ is true in $\pi_{\mathrm{n}}$ if $a \in a_{\mathrm{n}}$.

3. $\neg($ occurs $a)$ is true in $\pi_{\mathrm{n}}$ if $a \notin a_{\mathrm{n}}$

4. lasttime $p$ is true in $\pi_{\mathrm{n}}$ if $\mathrm{n}>0$ and $p$ is true in $\pi_{\mathrm{n}-1}$.

5. $\neg$ (lasttime $p$ ) is true in $\pi_{\mathrm{n}}$ if $\mathrm{n}=0$ or $\neg p$ is true in $\pi_{\mathrm{n}-1}$.

6. previously $q$ is true in $\pi_{\mathrm{n}}$ if $\mathrm{n}>0$ and for some $0 \leq j<n, q$ is true in $\pi_{\mathrm{j}}$.

7. $\neg$ (previously $q$ ) is true in $\pi_{\mathrm{n}}$ if $\mathrm{n}=0$ or for all $0 \leq j<n, \neg q$ is true in $\pi_{\mathrm{j}}$. 
8. $p$ before $q$ is true in $\pi_{\mathrm{n}}$ if for some $0 \leq j \leq n, p$ is true in $\pi_{\mathrm{j}}$, and for every $i \leq j, \neg q$ is true in $\pi_{\mathrm{j}}$.

9. $\neg(p$ before $q)$ is true in $\pi_{\mathrm{n}}$ if for every $0 \leq j \leq n$ such that $\neg p$ is not true in $\pi_{\mathrm{j}}$ (i.e., $\mathrm{p}$ could be true in an extension of $\pi_{\mathrm{j}}$ ) there is $i \leq j$ such that $q$ is true in $\pi_{\mathrm{i}}$.

10. $p$ since $q$ is true in $\pi_{\mathrm{n}}$ if for some $0 \leq j \leq n, q$ is true in $\pi_{\mathrm{j}}$, and for every $j \leq i \leq n, p$ is true in $\pi_{\mathrm{i}}$.

11. $\neg(p$ since $q)$ is true in $\pi_{\mathrm{n}}$ if for every $0 \leq j \leq n$, such that $\neg q$ is not true in $\pi_{\mathrm{j}}$, there is $j \leq i \leq n$ such that $\neg p$ is true in $\pi_{\mathrm{i}}$.

12. $p \wedge q$ is true in $\pi_{\mathrm{n}}$ if both $p$ and $q$ are true in $\pi_{\mathrm{n}}$.

13. $\neg(p \wedge q)$ is true in $\pi_{\mathrm{n}}$ if either $\neg p$ or $\neg q$ is true in $\pi_{\mathrm{n}}$.

14. $p \vee q$ is true in $\pi_{\mathrm{n}}$ if either $p$ or $q$ is true in $\pi_{\mathrm{n}}$.

15. $\neg(p \vee q)$ is true in $\pi_{\mathrm{n}}$ if both $\neg p$ and $\neg q$ are true in $\pi_{\mathrm{n}}$.

We can say that a formula $F$ is false in a path if $\neg F$ is true in the path, and $F$ is unknown if neither $F$ nor $\neg F$ is true in the path.

\subsection{Observations}

In addition to describing the laws that rule a dynamic system, which we do with an action description, we also need a way to express observations about the domain: what is true and what actions are occurring. The expressions used to record such observations are called observations or axioms, and are statements of the form: (1) initially $\lambda$, (2) $a_{e}$ occurs_at $t$, or (3) $\lambda$ observed_at $t$, where $\lambda$ is a literal, $a_{e}$ is an elementary or compound action, and $\mathrm{t}$ is a non-negative integer from [0..n]. Axioms of type (1) declare what is true in the initial state, axioms of type (2) state that "the elementary action $a_{\mathrm{e}}$ occurred at situation t", and axioms of type (3) state that "the literal $\lambda$ was observed to be true at situation $t$ ". The set of axioms that reflects the observations in a domain is referred to as the recorded history of the domain.

A particular instance of the system being described is given by a tuple formed by an action description and its recorded history, and is called a domain description.

\subsection{Queries}

One of the main purposes of a representation such as the one given in a domain description is to be able to extract answers to queries about a particular domain. A query in Alan is an expression of one of three types (1) $p$ holds at $\mathrm{t},(2) p$ holds in $\left[\mathrm{t}_{1}, \mathrm{t}_{\mathrm{k}}\right]$, or (3) $p$ after $\mathrm{A}$ at $\mathrm{t}$, where $p$ is a formula, $A$ is a sequence of actions, and $t, t_{1}$, and $t_{k}$ are either time points or $t_{c}$, denoting the current or most recent time point in history. 
Queries of type 1 ask if the given formula holds at the given time point. Queries of type 2 ask if the given formula holds over a period between time points $t_{1}$ and $t_{k}$. Queries of type 3 can be read as "if the sequence $A$ of user actions were executed at situation $\mathrm{t}$, would $\mathrm{p}$ hold afterward?".

By being able to model multimedia displays and answer such queries for a display specification, we can verify whether a resulting display indeed is the display intended by the user.

\section{MODELING MULTIMEDIA DISPLAYS}

Features of multimedia displays that can be modeled in Alan include:

- Navigation buttons that allow the user to go through different records in the database query answer.

- Displays with static data elements such as text and images.

- Displays with lists and triggers.

- Displays with multi-page documents (like e-books or PDFs).

- Displays with video and audio elements.

In general, to model a multimedia display system, its description is written as an action description in Alan. Objects that reflect particularities of the display are used as parameters for actions and fluents in predicates, and also in axioms when an actual multimedia document is modeled. The action description with particular recorded histories form a domain description that can then be used to formally verify the behavior of the display. The verification is done by queries that test the desired aspects of the behavior.

It is not our intention to reconstruct in a single framework the display features of all multimedia formats or displays. Rather we provide a metalanguage that can be used and adapted to suit the modeling of particular multimedia environments. Particularities of different environments are reflected through the use of objects. For example, if in the display being described one is using an xy-coordinate system to indicate the position of an element on the screen, then the object that represents that type of elements should include parameters for the $\mathrm{x}$ and $\mathrm{y}$ coordinates.

If the display being modeled is specified using a formal specification language, like SQL+D (Baral et al., 1998) then an automatic translation algorithm from the specification language into an action description in $\mathcal{A l a n}$ can be written. In this paper we do not assume a formal specification of the display, nor a particular layout paradigm, but assume a general description in English of the expected functionality of the multimedia display and a description of the schema of the database in question. This demonstrates the flexibility of our proposed method in modeling any multimedia display.

For each feature that needs to be modeled, we present the objects, actions, and fluents needed, as well as a set of predicates that reflect the behavior being modeled. 


\subsection{Modeling a multimedia tutorial}

As a typical example of the uses of multimedia database applications, we will model the multimedia training materials developed for the U.S. Corps of Engineers Research Laboratory land management (Cooper \& Smith, 2001). We will refer to it simply as the Peace Corps tutorial. It was developed independently from the modeling language presented here.

Elements in a database that include data and references to multimedia files (audio, video, images, animation) are used in the tutorial to compose pages. Pages result from a query and are built dynamically. A group of pages forms a unit, of which there are several in a training manual.

The interface has a two-level navigation system. Tabs displayed on top provide access to units. Within each unit, the user can navigate through pages using the buttons on the top left, as shown in Figure 1. The interface allows navigation to related material through hyperlinks in the Related Topics area, or to Keywords. Navigation through any of the methods provided changes the answer set shown in the display area.

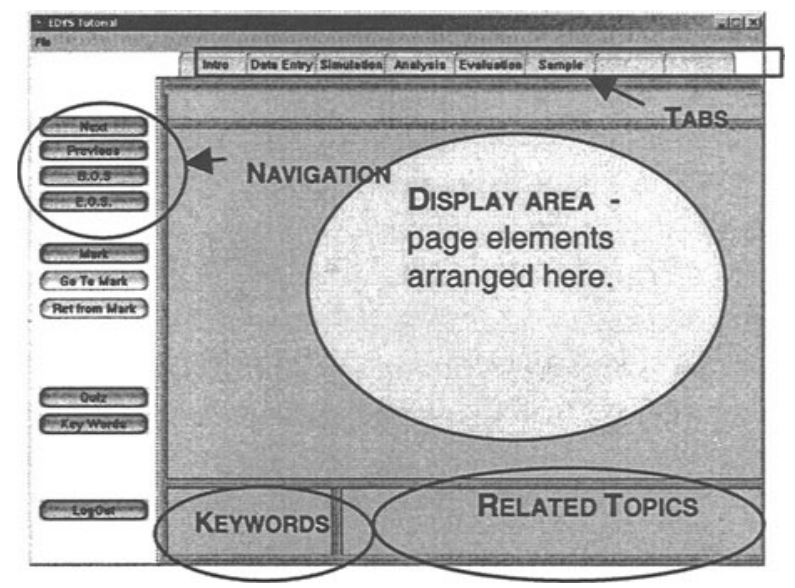

Figure 1. Display Environment of the US Corps of Engineers training manual.

\subsection{Modeling navigation buttons}

Almost all multimedia displays generated from database query answer include navigation buttons to go from record to record. Here we will model their behavior. 


\subsubsection{Objects, fluents, and actions}

Table 1 shows the basic set of constructs needed. Objects are introduced to the domain as an axiom using the fluent DECLARED.

Table 1. Basic constructs to model any display specification

\begin{tabular}{|c|c|c|}
\hline CONSTRICT & TYPE & DESCRIPTIO: \\
\hline $\operatorname{Panel}(\mathrm{u}, \mathrm{w}, \mathrm{x}, \mathrm{y}, \mathrm{V})$ & Object & $\begin{array}{l}\text { Panel } u \text { is located within panel } w \text { at } x, y \text { and has } \\
\text { content } V \text {. Parameter } w \text { is 'null' if } u \text { is the main } \\
\text { panel. } V \text { is a set of panels and/or display elements. }\end{array}$ \\
\hline Ctrl_Button(x,y) & Object & There is button $\mathrm{x}$ on panel $\mathrm{y}$. \\
\hline Answer_Set(R, x, Y, n) & Object & $\begin{array}{l}\mathrm{R} \text { is the relation or answer set currently being } \\
\text { displayed on area } \mathrm{x} \text {, its attributes are the set } \mathrm{Y} \text {, and } \\
\text { it contains } \mathrm{n} \text { records. }\end{array}$ \\
\hline ACTIVE_RECORD $(x, y)$ & Fluent & $\begin{array}{l}\text { Record } y \text { is the currently selected record from } \\
\text { answer set } x \text {. }\end{array}$ \\
\hline $\operatorname{DECLARED}(\mathbf{x})$ & Fluent & $\mathrm{x}$ is an object in the domain. \\
\hline $\operatorname{CLICK}(\mathrm{x}, \mathrm{y}, \mathrm{z})$ & Action & $\begin{array}{l}\text { The user "clicked" on } x \text {. Parameters } y \text { and } z \text { if } \\
\text { used, indicate particular occurrence of } x \text {, and a } \\
\text { particular record clicked. }\end{array}$ \\
\hline
\end{tabular}

As a general organizational element for the Peace Corps tutorial, we chose the object "Panel" to group of a display into logical units. The whole display area is referred to as the "main panel", and is also introduced via an object, Panel(main, NULL, $0,0 \mathrm{~V}$ ), where V is a set of ids of all other panels in it, including one for each of the five areas identified in Figure 1: navigation, tabs, display, keywords, and links.

In the navigation panel, there is an instance of the Ctrl_Button object for each of the navigation buttons, like Ctrl_Button(Next, navigation). These objects are included in all displays. If the answer set is a relation $\mathrm{R}$ obtained from an SQL Query that returns $\mathbf{n}$ records, the object Answer_Set would look as follows: Answer_Set(UnitPages, navigation, $\{$ PID $\}, n$ ).

\subsubsection{Action Description}

We model the behavior of navigation buttons with rules such as (P1).

(P1) CLICK(Ctrl_Button(Next, navigation)) causes ACTIVE_RECORD(R, i+1), $\neg \operatorname{ACTIVE} \_R E C O R D(R, z)$ if $\neg \mathrm{EQ}(\mathrm{z}, \mathrm{i}+1), \operatorname{ACTIVE} \_\operatorname{RECORD}(\mathrm{R}, \mathrm{i})$, DECLARED(Answer_set( $R$, navigation, ,, $\mathrm{n})$ ), LT(i,n)

(P1) can be read as follows: the action of clicking on the "Next" button causes the active record to become $i+1$ and not any other if the current active record is $i$ for the answer set to which the button is related, and $i$ is less than the total number of records in the answer set.

To "disable" a button when its action is not applicable, like for the "Next" button when we reach the last record, we use propositions like (P2). 
(P2) impossible CLICK(Ctrl_Button(Next, navigation)) if ACTIVE_RECORD(R, $\mathrm{n})$, DECLARED(Answer_Set(R, navigation,_,n))

\subsection{Displays with static elements}

Consider the display shown in Figure 2. Aside from the navigation buttons, we see an image and some text above it displayed in the display panel. These two elements are what we call "static": there is no timedependency associated to them, and actually not too much of a "behavior", aside from being "visible". We will use these elements to build our action description a-little bit further.

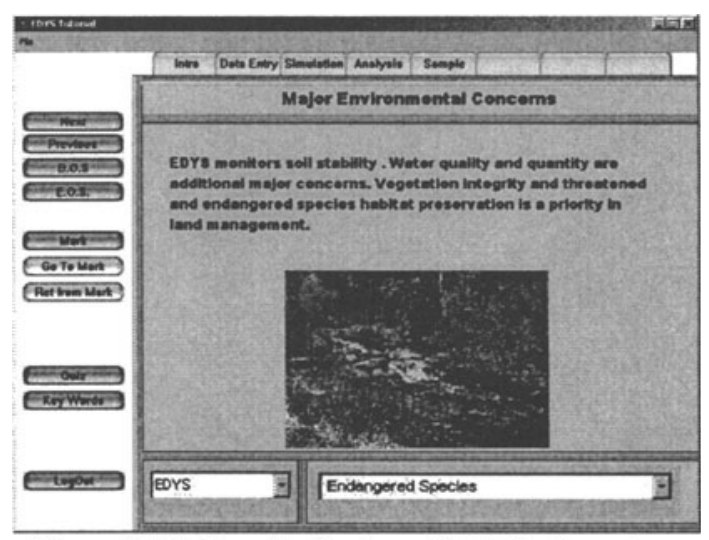

Figure 2. Multimedia display with static elements.

\subsubsection{Objects, fluents, and actions}

Table 2 shows the basic set of constructs needed to model the GUI elements of a display that are not time-dependant (static).

\subsubsection{Action Description}

The propositions in the action description to model these features deal mainly with determining what should be shown where. In multimedia documents we do not deal with the "when", since all elements are shown at the same time when the record becomes active. The timing at which each element becomes visible becomes important in multimedia presentations, which are outside the scope of this paper, presented in (Gonzalez 2000).

Each element included in a page is modeled by its corresponding instance of the "Disp_Element" object. For example, say an element of type "image" at position $(300,400)$ on the main screen, containing the file indicated in the attribute "Content" from the answer set $\mathrm{R}$, is identified as Img1. Its corresponding object would be: 
Disp_Element(Img1, image, Main, 300, 400, R.Content)

We now introduce some propositions to tell us which elements are visible. Consider Propostion (P3).

(P3) define $\operatorname{Visible}(R, i, j)$ if $\operatorname{ACTIVE} \_\operatorname{RecoRD}(R, j)$,

DeClaRED(Disp_element(y,t,p,_,_C)), BT(R.i, C), ᄀEQ(t, "list").

The above rule can be read "the value of record $j$, attribute $i$ of relation $R$ is visible if record $j$ is active, and there is a display element that includes it in its contents. Otherwise, the attribute is not visible". We exclude elements of type "list" from this rule.

Table 2. Constructs to model static components of a display.

\begin{tabular}{|c|c|c|}
\hline DONSTRLCT & TIPE & DESCRIPTIO: \\
\hline $\begin{array}{l}\text { Disp_Element( } u, v, w, x \text {, } \\
y, z)\end{array}$ & Object & $\begin{array}{l}\text { Display element } u \text { of type } v \text { is located in } w \text { at } \\
\text { position } x, y \text {, and its contents are } z \text {. }\end{array}$ \\
\hline $\operatorname{ISELEM}(\mathbf{x}, \mathbf{y})$ & Fluent & $\mathrm{x}$ is a display element of type $\mathrm{y}$ \\
\hline INCLUDES $(y, x)$ & Fluent & $\begin{array}{l}\mathrm{y} \text { includes, display element } \mathrm{x} \text {, directly or indirectly } \\
\text { (that is, } \mathrm{x} \text { might be inside a frame or panel). }\end{array}$ \\
\hline VISIBLE(R, y, z) & Fluent & $\begin{array}{l}\text { The value of attribute } y \text { of record } z \text { in answer set } R \\
\text { is visible. }\end{array}$ \\
\hline
\end{tabular}

\subsection{Modeling triggers and lists}

Triggers and lists are a very common feature in multimedia displays. This is similar to the behavior of navigation buttons, which trigger a change of the active record, but we need to expand the effects of such actions to model the launching of separate applications, or of derived displays (nested queries). For example, a button might link to a video file, and an application such as Real Player might be launched.

\subsubsection{Objects, fluents, and actions}

Table 3 shows the basic set of constructs needed to model displays with triggers and lists.

Table 3. Constructs needed to model triggers and lists.

\begin{tabular}{|c|c|c|}
\hline Constrite & Trpe & DESCRIPTION \\
\hline Trigger $(\mathbf{x}, \mathbf{y}, \mathbf{z})$ & Object & $\begin{array}{l}\text { Display element } \mathrm{x} \text { has a trigger which launches the query } \\
\text { or application y passing } \mathrm{z} \text { as parameters, where } \mathrm{z} \text { can be a } \\
\text { fixed value or an attribute of the database associated with } \\
\text { the display element. }\end{array}$ \\
\hline LAUNCHED(x,y) & Fluent & $\begin{array}{l}\text { External application y launched and is running as a } \\
\text { separate window from display element } x \text {. }\end{array}$ \\
\hline $\operatorname{PARAM}(x, y)$ & Fluent & $\begin{array}{l}\mathrm{y} \text { is a parameter list associated with display element } \mathrm{x} \\
\text { when a trigger is launched. }\end{array}$ \\
\hline DOUBLE_CLICK $(\mathrm{x})$ & Action & Like $C L I C K$. \\
\hline
\end{tabular}




\subsubsection{Action Description}

Proposition (P4) states that a double click on an element that has a trigger associated to it will cause the related query or application to be launched.

(P4) Double_Click ( $\mathrm{x}$ ) causes Launched(x,y) if Deflared(Trigger( $\mathrm{x}, \mathrm{y}, \mathrm{z})$ )

We can also model the parameters passed by using rules (P5) and (P6).

(P5) Param(y, $\left.R_{j}[A]\right)$ if DeClared(Trigger(x, y, z), Bt(R.A, z), ACtive_ReCoRd(R, j), $\operatorname{LAUNCHED}(x, y)$

(P6) Param(y, s) if Declared(Trigger( $x, y, z), B T(s, z)$, ISA_String(s), Launched( $x, y)$

Proposition (P3) determined which attribute values are visible for display elements other than lists. Proposition (P7) accounts for the fact that a list shows the associated attribute of all records at the same time.

(P7) define $\operatorname{Visible}(R, A, j)$ if DeClared(Disp_element $(y, t, z, \ldots,, V)$ ), INCLudes( $(p, z)$,

EQ(t, “list”), BT(R.A, V), DeCLARED(Answer_Set(R,p, , n)), GTE(j, 1), LTE(j,n)

In the Peace Corps tutorial, double clicking on an element of the list, like the Keywords or Related Topics lists, causes a new query to be triggered, displaying a new page. This is handled through a rule similar to (P4).

\subsection{Modeling a document display element}

A document display element is any that displays multi-page documents. Documents are similar to video and audio data elements in that they are composed of discrete elements ordered in a sequence that forms the whole. We call each of these constituent elements a "sample". Unlike the samples for video and audio files, a page in a document does not have a specified duration in time. It only changes when the user performs an action.

\subsubsection{Objects, fluents, and actions}

We introduce the notion of "occurrence" as a parameter in the fluents Current_Sample and Slider, allowing for multiple occurrences of the same display element. This additional parameter can easily be retrofitted into the rules previously presented. Table 4 shows the constructs needed.

Table 4. Constructs needed to model documents.

\begin{tabular}{|c|c|c|}
\hline Constr ("T & IVIl: & DRSTRIPTION \\
\hline Ctrl_Slider( $\mathrm{t}, \mathrm{x})$ & Object & Object $\mathrm{x}$ has a slider of type $\mathrm{t}$. \\
\hline SAMPLE_SIZE $(x, y)$ & Fluent & The size, in samples, of the attribute in $x$ is $y$. \\
\hline CURRENT_SAMPLE $(x, c, y, z)$ & Fluent & $\begin{array}{l}\mathrm{z} \text { is the sample of the file displayed on } \\
\text { occurrence } \mathrm{c} \text { of display element } \mathrm{x} \text { for record } \mathrm{y} \text {. }\end{array}$ \\
\hline SLIDE $(\mathrm{x}, \mathrm{c}, \mathrm{v})$ & Action & $\begin{array}{l}\text { Occurrence } c \text { of Ctrl_Slider object } \mathrm{x} \text { was dragged } \\
\text { to value } \mathrm{v} \text {, interpreted by the type of Slide } \\
\text { control. }\end{array}$ \\
\hline
\end{tabular}




\subsubsection{Action description}

Proposition (P8) shows a rule that can be read "clicking on the Previous page button causes the current sample to become $s-1$ if the current sample is $s$ and $s$ is not 1", in which case the action would be declared impossible (the button is disabled). Similar rules would be added for the Next button and simpler ones for the First and Last buttons of the document display element.

(P8) CLICK(Ctrl_Button(PrevPage, $x$ ), c) causes CuRRENT_SAMPLE( $x, c, j, s-1$ ),

$\neg$ CURRent_SAmple $(x, c, j, z)$ if $\neg$ EQ(z, s-1), CurRent_Sample(x, c, j, s), GT(s, 1),

ACTIVE_ReCORD(R, $\mathbf{j})$

Proposition (P9) states that by default, the first page of the document is the current sample when the record becomes active for the first time. Otherwise, the sample it was at in the previous visit is the current sample.

(P9) Current_Sample $(x, c, j, 1), \neg$ CURRENT_Sample $(x, c, j, z)$ if $\neg E Q(z, 1)$,

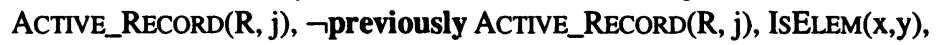
EQ(y, "document") $\vee$ EQ(y, "video") $\vee$ EQ(y, "audio")

The same rule applies to video and audio display elements. This proposition is the first one to use a temporal operator, "previously", and is the introduction of the non-Markovian aspects of the model.

The initial axiom set for such a display would include all the DECLARED fluents for the different Disp_element objects in the different panels. Consider adding the following observations to the initial axiom set :

SAMPLE_SIZE $(B, 105)$ observed_at 0

CLICK(Ctrl_Button(NextPage, doc)) occurs_at 1

CLICK(Ctrl_Button(Next, navigation)) occurs_at 2

SAMPLE_SIZE $(B, 30)$ observed_at 3

CLICK(Ctrl_Button(Previous, navigation)) occurs_at 3

SAMPLE_SIZE $(B, 105)$ observed_at 4

These axioms are observations that reflect what is happening on the screen: on the first record, the user looked at page 2, then went to the next record, and then back to the first. The axioms, in combination with the action description, form a domain description, and allow us to ask queries about the behavior of the above display. We can verify with a simple query that when returning to the first record, page 2 will be displayed:

CURRENT_SAMPLE(Doc1, 1, 1, 2) holds_at 4

The proof is outside the scope of this paper, appears in (Gonzalez, 2000).

\subsection{Video and audio files}

We want to model the general contents and behavior of the display at particular points in time. In displays that include audio, video, and temporal presentations, display elements change constantly. We want to define "points in time" in this continuum that will reasonably represent the display 
contents. If the frame rate of a video were six frames per second, we should be able to represent every time a new frame is shown, that is, every $1 / 6$ of a second. A smaller granularity would serve no practical purpose, since the display does not change within that interval. For audio files it is not practical to try to refer to each sampling, since sampling rates are very small. Usually, sound samplings are given in seconds and fractions of a second.

\subsubsection{Objects, fluents, and actions}

Table 5 shows the set of constructs needed to model video and audio.

Table 5. Constructs needed to model triggers and lists.

\begin{tabular}{|c|c|c|}
\hline CoNsthI CT & TYPE & Dese ription \\
\hline $\begin{array}{l}\text { STOPPED }(x, c) \\
\text { PLAYING }(x, c) \\
\text { PAuSED }(x, c)\end{array}$ & Fluent & $\begin{array}{l}\text { The occurrence } \mathrm{c} \text { of display element } \mathrm{x} \text { is Stopped, Playing, } \\
\text { or Paused }\end{array}$ \\
\hline $\operatorname{CLOCK}(x, c, i, y)$ & Fluent & $\begin{array}{l}\text { The occurrence } \mathrm{c} \text { of display element } \mathrm{x} \text { for record } \mathrm{j} \text { has clock } \\
\text { value } \mathrm{y} \text {. }\end{array}$ \\
\hline $\begin{array}{l}\text { SAMPLING_RATE } \\
(\mathrm{x}, \mathrm{r})\end{array}$ & Fluent & $\begin{array}{l}\text { The sampling rate for a video or audio object displayed in } \mathrm{x} \\
\text { is } \mathrm{r} \text { frames per second, in the case of videos, or } \mathrm{r} \mathrm{kHz} \text {, in the } \\
\text { case of audio files. }\end{array}$ \\
\hline $\begin{array}{l}\text { RUNNING_TIME } \\
(x, t)\end{array}$ & Fluent & $\begin{array}{l}\text { The time } t \text { in seconds is the running time for a video or } \\
\text { audio object displayed in display element } \mathbf{x} \text {. }\end{array}$ \\
\hline CLOCK_TIC (t) & Action & $\begin{array}{l}\text { An action that occurs every time the system time advances } t \\
\text { milliseconds. }\end{array}$ \\
\hline
\end{tabular}

\subsubsection{Action description}

Consider the screen from the Peace Corps application where only a video is shown with some control buttons for allowing user interaction while it is playing: Play, Stop, and Pause. We use instances of the Ctrl_Button object. For example, Ctrl_Button("Play", $x$ ) will associate a "Play" button to display element $\mathrm{X}$. PAUSED, STOPPED, and PLAYING give the status of the video or audio display elements, and are mutually exclusive. Propositions such as (P10) model the effect of clicking on the buttons, in this case, "Play". (P10)CLICK(Ctrl_Button(“Play", $x), c$ ) causes PlAYING( $x, c), \neg \operatorname{STOPPED}(x, c)$, $\neg \operatorname{PAUSED}(\mathrm{x}, \mathrm{c})$

The fluent CLOCK is a timer set for a particular occurrence of a display element. It takes the id of the display element in question, plus an identifier of the particular occurrence of the display element, the record number, and a value as parameters. The behavior of the clock of a particular display element in response to different events, like passing time or the user sliding forward to a later point in the video, is modeled using propositions such as (P11), which shows that the value of the clock is set to zero when the "Stop" button is pressed, and proposition (P12), which shows how the system action 
CLOCK_TIC causes an increment in the value of the CLOCK fluent for all display element that are PLAYING. For clarity, we are not including in the rules the fact that the clock value is unique, but it is assumed.

(P11) CuICK(Ctrl_Button(“Stop", $x$ ), c) causes Clock( $x, c, j, 0)$ if ACtive_ReCORD( $R$,

j), DECLARED(Answer_Set(R, navigation,_,n))

(P12) CLOCK_TIC (t) causes Clock $(x, c, j, y+t)$ if Clock $(x, c, j, y), \operatorname{Playing}(x, c)$,

ACTIVE_RECORD(R, j), DECLARED(Answer_Set(R, navigation,_, $n)$ )

There is also a rule that reflects the effects of the action SLIDE, which changes the value of the fourth parameter of Clock directly, setting it to the point in time where the Slide is released. The fluents SAMPLING_RATE, RUNNING_TIME, and SAMPLE_SIZE characterize the particular video or audio file. Based on any two of them and the CLOCK fluent, propositions determine the current sample. Proposition (P13) shows that the CLOCK value of a particular element is greater than or equal to its running time, the status of the element becomes "Stopped".

(P13) $\operatorname{Stopped}(x, c), \neg \operatorname{Playing}(x, c), \neg \operatorname{Paused}(x, c)$ if $\operatorname{IsElem}(x, y), E Q(y$, "video") $v$ EQ(y, "audio"), Playing(x, c), ACtive_ReCORD(R, j), DEClaRed(Answer_Set(R, navigation,_,n)), CLOCK(x, c, j, t), RUNNING_Time(x, T), GEQ(t, T)

The current sample is calculated by multiplying the corresponding CLOCK value by the sampling rate of the attribute being shown.

Other propositions specify that the status of a video or audio elements is "Stopped" by default when the record becomes active for the first time. Similarly, and illustrating the use of the non-Markovian features of the modeling language, (P14) specifies that the status of a video or audio element is "Paused" when the record that contains it has been active before and just became active again. Just as we did for the document display element, the first sample showed would be the one last seen when the user left the record. The current sample would be calculated to be the same since the Clock does not change while the record is not active.

$$
\begin{aligned}
& \text { (P14) PAused(x, c), } \neg \text { Playing(x, c), } \neg \operatorname{STOPPED}(x, c) \text { if IsElem(x, y), EQ(y, "video") } v \\
& \text { EQ(y, "audio"), ACtive_ReCORD(R, j), DECLARED(Answer_Set(R, navigation,_,n)) } \\
& \text { lasttime } \neg \text { ACTIVE_ReCORD(R, j), previously ACTIVE_ReCORD(R, j) }
\end{aligned}
$$

\section{RELATED WORK}

To our knowledge, the only other direct attempt at constructing formal semantics of dynamic displays is that by (Scherl et al., 1998), which presents a Situation Calculus model of Hypertext. However, the features are limited to modeling the dynamic aspects of link traversal and the functionality of buttons. As for the non-Markovian action language, this approach is also unique to the best of our knowledge. (Gabaldon, 2000) presents a nonMarkovian extension to Situation Calculus, which is more limited than action languages. A more extensive analysis of related work is presented in the full version of this paper and in (Gonzalez, 2000). 


\section{REFERENCES}

Gonzalez, Graciela (2000), An SQL extension for specification of multimedia displays: its syntax, architecture, and semantics based on a non-Markovian action language. Dissertation. University of Texas at El Paso.

Baral, C., Gelfond, M., and Provetti, A. (1997), "Representing Actions: Laws, Observations and Hypotheses", The Journal of Logic Programming, 31(1-3):201-244.

Watson, Richard (1999), Action Languages and Domain Modeling, Doctoral Dissertation, University of Texas at El Paso.

Baral, C., Gonzalez, G., and Nandigam, A. (1998) "SQL+D: Extended Display Capabilities for Multimedia Database Queries" Proceedings of ACM's Multimedia 98, ACM, September, 1998.

Scherl R., Bieber M., and Vitali F. (1998) "A Situation Calculus Model of Hypertext" Proceedings of the 31st Hawaii International Conference on System Sciences, IEEE Press

Cooper, P.A., and Smith G.W. (2000) A Development of a Hydrology Data Repository and Models Tutorial, Project funded through U.S. Corps of Engineers Research Laboratory and the Texas Research Institute for Environmental Science.

Gabaldon, Alfredo (2000) "Non-Markovian Control in the Situation Calculus", The Second International Workshop on Cognitive Robotics (held in conjunction with ECAI2000).

Shanmugan, S. and Breipohl, A.M.(1988) "Markov Sequences and Processes", Random Signals: Detection, Estimation, and Data Analysis, John Wiley \& Sons.

\section{BIOGRAPHIES}

Graciela Gonzalez is an Assistant Professor of Computer Science at Sam Houston State University. She received his M.S. (1994) and $\mathrm{PhD}$ (2000) in Computer Science from the University of Texas at El Paso. During the course of her studies, she was the main author to published papers in conferences such as ACM Multimedia '98, IEEE's ICDE '98 and ICMCS '99, among others, and worked as a Software Engineer and Multimedia Development Manager. Her main research interests include multimedia databases, human-computer interaction, theories of actions and logic programming.

Chitta Baral is an associate professor at the Arizona State University. He obtained his B.Tech(Hons) degree from the Indian Institute of Technology, Kharagpur and his M.S and Ph.D degrees from the University of Maryland at College Park. He has been working in the field of knowledge representation and logic programming since 1988, and his research has been supported over the years by National Science Foundation, NASA, and United Space Alliance. He received the NSF CAREER award in 1995 and led successful teams to AAAI 96 and 97 robot contests. He has published more than 65 articles in Logic Programming, Knowledge Representation, and Artificial Intelligence conferences and Journals.

Peter Cooper is an Associate professor of Computer Science at Sam Houston State University. He received his M.A.(1990) and Ph.D.(1993) from the University of MissouriColumbia. His main research interests include two and three dimensional imaging and human computer interaction. 